\title{
A New Approach for Fairness Increment of Consensus-Driven Group Recommender Systems Based on Choquet Integral
}

\author{
Cu Nguyen Giap, Graduate University of Science and Technology, Vietnam \\ (iD) https://orcid.org/0000-0002-2189-0036
}

Nguyen Nhu Son, Institute of Information Technology, Vietnam Academy of Science and Technology, Vietnam* Nguyen Long Giang, Institute of Information Technology, Vietnam Academy of Science and Technology, Vietnam (iD) https://orcid.org/0000-0001-6184-1469

Hoang Thi Minh Chau, University of Economics Technology for Industries (UNETI), Hanoi, Vietnam Tran Manh Tuan, Thuyloi University, Hanoi, Vietnam Le Hoang Son, VNU Information Technology Institute, Vietnam National University, Hanoi, Vietnam (iD) https://orcid.org/0000-0001-6356-0046

\begin{abstract}
Group recommender systems (GRSs) in most e-commerce and tourism applications like Booking. com, Traveloka.com, Amazon, etc. have risen in recent years. One of the most concerning problems in GRSs is to guarantee the fairness between users in a group called the consensus-driven group recommender system. This paper proposes a new flexible alternative that embeds a fuzzy measure to aggregation operators of consensus process to improve fairness of group recommendation and deals with group member interaction. Choquet integral is used to build a fuzzy measure based on group member interactions and to seek a better fairness recommendation. The empirical results on the benchmark datasets show the incremental advances of the proposal for dealing with group member interactions and the issue of fairness in consensus-driven GRS.
\end{abstract}

\section{KEYWORDS}

Choquet Integral, Fairness Increment, Fuzzy Measure, Group Recommender System

\section{INTRODUCTION}

Group recommender system (GRS) becomes a crucial tool to develop an information system supplying recommendation to joined activity of group (Dara et al., 2020). GRSs can be categorized into two common approaches in which the former creates a group profile by merging those of all members and uses it as a pseudo single user in the process of recommendation (Da'u \& Salim, 2020). The later called consensus phase generates all group members' preferences and aggregates them to select the suitable recommendation to group (Kuhlman \& Rundensteiner, 2020; Banda et al., 2020). 
One of the most concerned problems in GRSs is to guarantee the fairness between users in a group so-called the consensus-driven group recommender system. This approach makes recommendation for the group based on individual interests using consensus mechanisms. It utilizes the information of individuals, and a set of preferred items recommended to a group based on individuals' sets of preferred items. The fairness issue in GRSis a social concept and not easy to measure(Kaya, Bridge \& Tintarev, 2020). Recently, several researchers have defined the fairness in GRS explicitly as the ratio of satisfied people to total group members (Felfernig et al., 2018), the equity of group members satisfactions (Xiao et al., 2017) or consider fairness of recommended item set as a package rather than a set of independent items (Serbos et al., 2017). Nevertheless, a challenge raised in finding good fair solution in consensus-driven GRS is that a member preference for an item is often influenced by members interaction and item relations (Wang et al., 2017). Therefore, it is difficult to estimate the imbalance between group members' preferences when regarding members' interactions.

When dealing fairness issue, the common GRSs do not deal with effect of interaction between group's members. In this case, the user-item relevance do not change when group members change, thus the fairness of recommendation is simply calculated from individual preferences (Dara et al., 2020). On the other hand, a number of studies presented solutions for estimating group preference regarding group member relationship from external information such as the user social network profile (Yin et al., 2020) or internal information from the group member distances (Castro et al., 2015). However, these studies did not stress on the fairness problem in GRSs as expected.

In order to cope the fairness issue, we are motivated by the aggregation operators in the consensus phase of GRS. That is to say, if a well-defined aggregation operator is formed, we can achieve the fairness between users in a group while still maintaining reasonable accuracy of prediction and recommendation. There are many aggregation operators presented in the literature to generate group preference such as the additive utilitarian strategy, average strategy, least misery strategy, approval voting strategy, fairness strategy, and so on (Cantador \& Castells, 2012). Several strategies maximize the total group preference such as additive utilitarian strategy, average strategy and other strategies promote weaken member such as least misery strategy, Borda Count strategy, approval voting strategy, fairness strategy. However, these strategies do not solve fairness issue of group RS directly, even fairness strategy does not deal with the imbalance of satisfactions between group members.

In this paper, a new flexible framework is proposed to deal with both fairness issue and user interaction meanwhile maintain major objective of consensus-driven GRS that is finding high accumulative group members' satisfaction. Instead of the traditional aggregation techniques, Choquet integral is use to generate group recommendation in the consensus phase (Huynh et al., 2020). The Choquet integral based aggregation extends weighted aggregation to give a balanced recommendation through capacity functions(Lust, 2015). The proposed consensus-driven GRS includes two phases: recommendation and consensus phase. In the recommendation phase, individuals' preferences are recommended using the user-based collaborative filtering while the consensus phase calculates the group preference by Choquet integral-based aggregation. The contributions of this paper are several new approaches for generation of capacity function such as reducing the imbalance between members(a.k.a. pure fairness-aware capacity function) and considering more influence of user interactions to user satisfaction in community (a.k.a. adjusted-fairness capacity function). The system is evaluated on the MovieLens-1M dataset by different metrics such as mean absolute error (MAE), group preference metric, and fairness metrics.

The next sections will present the background and the proposed consensus-driven GRS based on Choquet integral. Sections 4 and 5 show the experimental results and the conclusion with future works. 


\section{PRELIMINARY}

\subsection{Consensus-Driven GRS}

The consensus-driven GRS includes two phases: recommendation and consensus. In the recommendation phase, individuals' preferences are created using traditional filtering techniques of recommender systems such as user-user collaborative filtering. The consensus phase generates recommendation to group by aggregating individuals interests (Castro et al., 2015). The consensus phase concept is depicted in Fig.1.

Figure 1. The consensus phase in GRS

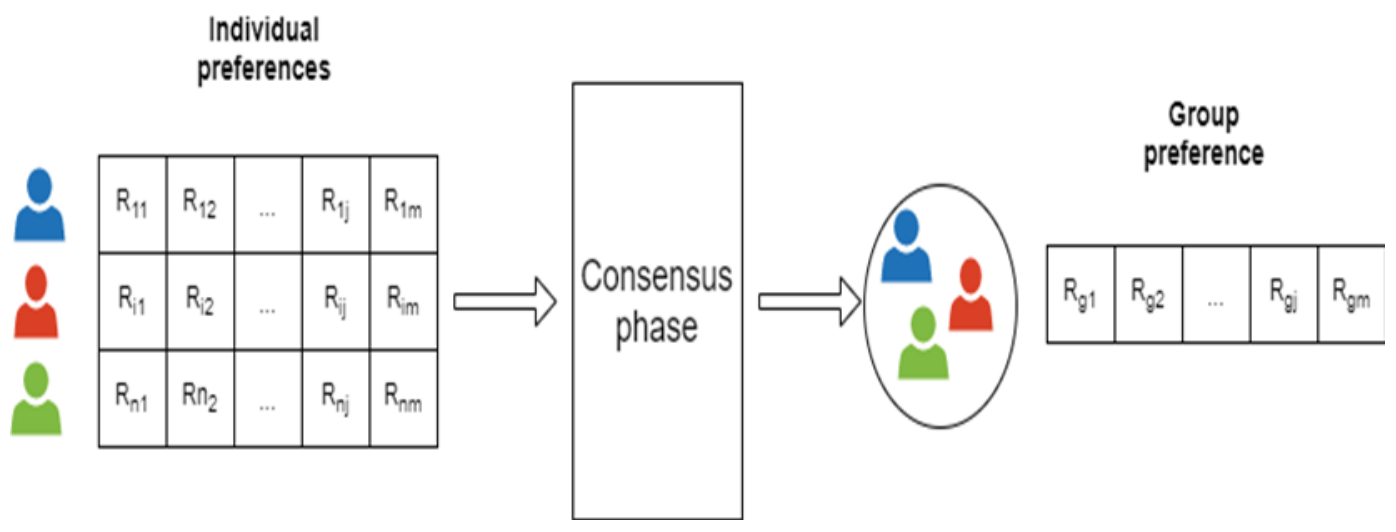

In the consensus phase, several aggregation approaches have been presented such as,

Additive utilitarian strategy (AUS):calculates group preference on an item by,

$\operatorname{pref}_{i}^{g}=\sum_{u \in g} r_{u, i}$

where $r_{u, i}$ is the rank that a user $u$ of group $g$ gives for item $i$.

Multiplicative strategy (MS):instead of additive, this strategy calculates group preference on an item by multiply user preferences,

$$
\operatorname{pref}_{i}^{g}=\prod_{u \in g} r_{u, i}
$$

Least Misery Strategy (LMS): group preference is equal to user preference who gives lowest rank.

$\operatorname{pref}_{i}^{g}=\min _{u \subset g}\left(r_{u, i}\right)$

Most Pleasure Strategy (MPS): group preference is equal to user preference who gives highest rank. 


$$
\operatorname{pref}_{i}^{g}=\max _{u \in g}\left(r_{u, i}\right)
$$

Approval Voting strategy (AVS): group preference is equal to number of users voted to one item.

$$
\operatorname{pref}_{i}^{g}=\sum_{u \in g} v_{u, i}
$$

where, $v_{u, i}$ is 1 if user $u$ vote to item $i$ and is 0 otherwise.

Copeland Rule strategy (CRS): inspired by Copeland index, group consensus phase selects items based on the time that an alternative beat or lose to other alternatives.

$$
\operatorname{pref}_{i}^{g}=\sum_{j \in I} c_{i, j}
$$

where $c_{i, j}$ is equal to 1 if item $i$ beats item $j$. It means that the number of users rank $i$ higher than $j$ is greater than the opposite. The value of $c_{i, j}$ is -1 if $j$ beats $i$ and $c_{i, j}$ is 0 in other situations.

Borda Count strategy (BCS): replaces the rank that a user gives for an item set by new award using the Borda count. Group preference is calculated by additive strategy. The new award of each item is its position in user individual preference list, and this list is index from zero.

Fairness strategy $(\boldsymbol{F S})$ : This strategy selects a user in group one by one, and the selected user chooses the best item for him. This process repeats until the number of chosen items reaches the need.

Regarding the complexity of all strategies, calculating the preference a group $g$ for item $i$ using different aggregation operators is the same $\mathrm{O}(|g|)$. The complexity of consensus mechanisms is $\mathrm{O}(|I| \cdot|g| . N)$ where $N$ is the number of top items need to recommend. Meanwhile, the Copeland Rule s and Borda Count need to compare between all items to calculate the preference of a group $g$ for item $i$, so that the complexity is $\mathrm{O}(|g| \cdot|I|)$. The consensus mechanisms' complexity is $\mathrm{O}\left(|I|^{2} \cdot|g| \cdot N\right)$, respectively.

\subsection{Fairness in Group Recommender Systems}

In order to maximize the utility of recommender systems, in general, many researches tried to improve the fairness of recommendation. In the case that a single item is recommended to a group, the fairness of such recommendation would be specified by the number of members who satisfy with recommendation. The satisfaction is defined by member's preference related to a threshold (Felfernig et al., 2018).

$$
\operatorname{fairness}(g, i)=\frac{\left|U_{u \in g} r_{u, i} \geq t h\right|}{|g|}
$$

This definition of fairness is the ratio of the satisfied members and the number of group member. It can be extended for a set of items by using an average operator over all items. A satisfied member is a user who rated an item at a rank equal or greater a threshold th .GRS has multi-stakeholders, and fairness element is a factor presents the imbalance of satisfactions of members as a recommendation 
given to a group. When a set of items is recommended to a group of people, there are different points of view about fairness that is a measurement of the imbalance of users satisfy recommendation.

According to (Xiao et al., 2017), fairness presents the imbalance between satisfactions of all members of a group. Let $S(u, I)$ is a measure of satisfaction of user $u$ with a set ofrecommended items $I$. Then, the fairness of recommendation $I$ to a group of users $g$ can be calculated by following alternatives.

Least misery:

$$
\text { fairness }_{L M}(g, I)=\min (S(u, I), \forall u \in g)
$$

Variance:

$$
\text { fairness }_{V a r}(g, I)=1-\operatorname{Var}\{S(u, I), \forall u \in g\}
$$

Jain's Fairness:

$$
\text { fairness }_{\text {Jain }}(g, I)=\frac{\left(\sum_{u \subset g} S(u, I)\right)^{2}}{|g|^{*} \sum_{u \subset g} S(u, I)^{2}}
$$

Min-Max ratio:

$$
\text { fairness }_{\text {min-max }}(g, I)=\frac{\min (S(u, I), \forall u \in g)}{\max (S(u, I), \forall u \in g)}
$$

The satisfaction of a user on a set of items is calculated by,

$$
S(u, I)=\frac{\sum_{i \subset I} r_{u, i}}{|I|}
$$

The authors also introduced and compared performance of some Greedy-based algorithms regarding the fairness of the GRS (Xiao et al., 2017).

Serbos et al. (2017) proposed two proportional fairness estimators. The first one uses the definition of $m$ - proportional of a package of items, which presents to any pakage that has at least $m$ items user $u$ like. Then, fairness is defined as follow.

$$
\text { fairness }_{\text {m-propotional }}(g, I)=\frac{\left|U_{I}\right|}{|g|}
$$


where $U_{I}$ is the set of users having package $I$ is a $m$-proportional .

The second approach uses the definition of $m$-envy - freeness that shows the number of items favoured by a user $u$ that are among top preference of other members.

fairness $_{m-\text { envy-freeness }}(g, I)=\frac{\left|U_{I}\right|}{|g|}$

where $U_{I}$ is the set of users that have package $I$ is a $m$-envy-freeness .

\subsection{Choquet Integral and Fuzzy Measure}

a. Capacity Function

In a multi-criteria decision-making problem, with $œ$ criteria:

Definition 1: A capacity is a set of function $\mu: 2^{\mathrm{M}} \rightarrow[0,1]$ such that

$$
\mu(\varnothing)=0, \mu(\mathrm{M})=1 \text { (boundary condition) }
$$

$\forall A, B \subset \mathrm{M}$, such that $A \subseteq B, \mu(A) \leq \mu(B)$ (monotonicity condition)

Capacity presents the role of a subset $A$ of criteria set $\mathrm{M}$.

Definition 2: A capacity is said additive if for each pair of subsets ' ,' $\in \mathcal{M}$, $\mu(A \cup B)=\mu(A)+\mu(B)$, other while, a capacity is a non-additive.

b. Choquet Integral

Definition 3: The Choquet integral of a vector $r$ in the $\mathrm{m}^{\text {th }}$ dimension with respect to a capacity $\mu$ is defined by:

$$
C Q_{\mu}(r)=\sum_{i=1}^{m}\left(r_{i}^{\uparrow}-r_{i-1}^{\uparrow}\right) \mu\left(\Upsilon_{i}^{\uparrow}\right)
$$

where $r^{\uparrow}=\left\{r_{1}^{\uparrow}, \ldots, r_{m}^{\uparrow}\right\}$ is a permutation of the elements of $r$ with $0=r_{0}^{\uparrow} \leq r_{1}^{\uparrow} \leq \ldots \leq r_{m}^{\uparrow}$, and the set $\Upsilon_{i}^{\uparrow}=\left\{j \in \mathrm{M}, r_{j} \geq r_{i}^{\uparrow}\right\}=\left\{i^{\uparrow},(i+1)^{\uparrow}, \ldots, m^{\uparrow}\right\}$ for $i<m$ and $\Upsilon_{m+1}^{\uparrow}=0$.

c. Choquet Optimal Set

Definition 4: Given a set $\Gamma$ of alternatives, the Choquet optimal set, called $\Gamma_{C}$, is the set has all optimal alternatives, for each possible Choquet integral, that is $\forall \mu \in \Upsilon, \exists r^{i} \in \Gamma_{C}\left|C Q_{\mu}\left(r^{i}\right) \geq\right| C Q_{\mu}\left(r^{j}\right) \forall r^{j} \in \Gamma$, where $\Upsilon$ is a set of all possible capacity defined over set of criteria $M$.

Lust(2015)assessed the possibility of reaching a Choquet optimal alternative by Choquet integral using a capacity function generated randomly with a uniform law. This test was performed under the multi-criteria decision contexts, and the authors argued that random generation of fuzzy measure with 
a uniform law works well when the number of criteria greater than 5. Even this test was only done with the number of criteria smaller than 10 , it proved the potential power of Choquet integral-based aggregation over the weight sum aggregation.

\section{d. Random Generation of Fuzzy Measure}

Generating a non-additive capacity (or fuzzy measure) randomly is an interesting and useful researching area, and in this part, we are going to review the state of the arts in this area and its complexity to assess the application possibility of these methods in context of GRS. According to the definition of the capacity function, the number of coefficients must be estimated is $2^{|\mathrm{M}|}-2$ with $|\mathrm{M}|$ criteria, and it cannot be done completely with large number of criteria because the complexity grows exponentially. However, in order to apply Choquet-integral, the problem of identifying a complete capacity function reduces to identifying all values of partial order set (poset in short) $\wp(\mathrm{M}, \leq)$ (Combarro et al., 2013).

One of the most computing expensive processes in an exact uniform random generator is generating all linear extensions from a poset. The best approach for this issue was introduced in (Lerche \& Sørensen, 2003) with time complexity as $\bigcirc\left(n^{5} \log n\right)$ where $n$ is the number of elements in a partial order set. Even these heuristic algorithms improve the speed of random generation of linear extensions, they cannot be applied in the multi-criteria decision with a large number of criteria (Lust, 2015).

\section{THE PROPOSED CONSENSUS-DRIVEN GRS BASED ON CHOQUET INTEGRAL}

In order to apply Choquet integral-based aggregation in GRS, it is required to compute the capacity function for each group of users. Therefore, it is impossible to generate capacity function randomly due to computing expensive. The feasible approach is proposing an algorithm that evaluate values of points in needed linear extensions directly, and it must be a computing efficient process. This section follows this approach and defines several capacity functions based on user interaction and fairness objective of a GRS.

\subsection{Why Using Choquet Integral in Consensus-Driven GRS?}

In formal approaches for consensus phase, additive utilitarian strategy seems to be a regular choice because it maximizes the total individual's preferences. However, fairness has become more important than the additive preference. Applying Choquet integral can improve fairness issue while regarding the objective of additive preference. An encouraged example is present below.

Example 1: The tables below present the ranks of three items given by three users with different approaches for consensus phase.

Table 1. Simple additive utilitarian approach

\begin{tabular}{|l|l|l|l|}
\hline Users Items & $\mathbf{I}_{\mathbf{1}}$ & $\mathbf{I}_{\mathbf{2}}$ & $\mathbf{I}_{3}$ \\
\hline $\mathbf{U}_{\mathbf{1}}$ & 10 & 4 & 3 \\
\hline $\mathbf{U}_{\mathbf{2}}$ & 1 & 9 & 8 \\
\hline $\mathbf{U}_{\mathbf{3}}$ & 10 & 5 & 2 \\
\hline Group's preference & $\mathbf{2 1}$ & $\mathbf{1 8}$ & $\mathbf{1 3}$ \\
\hline
\end{tabular}

Group preference order: $\left|I_{1}>\right|_{2}>\left.\right|_{3}$. 
This is also the same result for the average strategy approach.

Consensus with Choquet integral-based aggregation approach: Let $\mu$ being the capacity function defined by:

$$
\begin{aligned}
& \mu\left(\left\{U_{1}\right\}\right)=\mu\left(\left\{U_{2}\right\}\right)=\mu\left(\left\{U_{3}\right\}\right)=0.2 ; \mu\left(\left\{U_{1}, U_{2}\right\}\right)=\mu\left(\left\{U_{2}, U_{3}\right\}\right)=\mu\left(\left\{U_{1}, U_{3}\right\}\right)=0.4 \text {; and } \\
& \mu\left(\left\{U_{1}, U_{2}, U_{3}\right\}\right)=1 .
\end{aligned}
$$

The Choquet-based aggregation of item's preference is set as follows:

$\operatorname{pref}_{I_{1}}^{C Q}=(1-0) \mu\left(\left\{U_{1}, U_{2}, U_{3}\right\}\right)+(10-1) \mu\left(\left\{U_{1}, U_{3}\right\}\right)+(10-10) \mu\left(\left\{U_{3}\right\}\right)=1+9 * 0.4=4.6$

$\operatorname{pref}_{I_{2}}^{C Q}=(4-0) \mu\left(\left\{U_{1}, U_{2}, U_{3}\right\}\right)+(5-4) \mu\left(\left\{U_{2}, U_{3}\right\}\right)+(9-5) \mu\left(\left\{U_{2}\right\}\right)=4+1^{*} 0.4+4^{*} 0.2=5.2$

$\operatorname{pref}_{I_{3}}^{C Q}=(2-0) \mu\left(\left\{U_{1}, U_{2}, U_{3}\right\}\right)+(3-2) \mu\left(\left\{U_{1}, U_{2}\right\}\right)+(8-3) \mu\left(\left\{U_{2}\right\}\right)=2+1 * 0.4+5 * 0.2=3.4$

\section{Table 2. The order of items}

\begin{tabular}{|l|l|l|l|}
\hline UsersIItems & $\mathbf{I}_{\mathbf{1}}$ & $\mathbf{I}_{2}$ & $\mathbf{I}_{\mathbf{3}}$ \\
\hline $\mathbf{U}_{\mathbf{1}}$ & 10 & 4 & 3 \\
\hline $\mathbf{U}_{\mathbf{2}}$ & 1 & 9 & 8 \\
\hline $\mathbf{U}_{\mathbf{3}}$ & 10 & 5 & 2 \\
\hline Group's preference & $\mathbf{4 . 6}$ & $\mathbf{5 . 2}$ & $\mathbf{3 . 2}$ \\
\hline
\end{tabular}

Group preference order: $\mathrm{I}_{2}>\mathrm{I}_{1}>\mathrm{I}_{3}$

It can be seen that the item $\mathrm{I}_{2}$ is the most balancing item. The above example motivates the use of Choquet-integral operation to expand the additive aggregation process of consensus phase to reach a more balancing alternative.

\subsection{The Proposed Consensus-Driven GRS Method}

The proposed consensus-driven GRS method includes two phases below:

- The recommendation phase: individuals' preferences are recommended using the user-based collaborative filtering technique.

- The consensus phase: group preference is calculated through Choquet integral-based aggregation.

In the recommendation phase, User-based collaborative filtering is a useful technique to predict a user preference on an item that is not ranked. This technique includes two steps. The first step identifies a set of user's neighbours share some characteristics with the target user. The distance between users is estimated by similarity metrics. Pearson similarity and Cosine similarity are two well-known measures. However, in this study we only investigate the performance of Cosine similarity below,

$$
\operatorname{sim}_{\cos i n}(a, b)=\frac{\sum_{i \subset I} r_{a, i} r_{b, i}}{\sqrt{\sum_{i \subset I} r_{a, i}^{2}} \sqrt{\sum_{i \subset I} r_{b, i}^{2}}}
$$

where, $I$ is set of items ranked by both users $a, b$.

The next step is generating the predicted user preference on an item with unknown rank. 


$$
r_{a, j}=\bar{r}_{a}+\alpha \sum_{u \subset U} \operatorname{sim}(a, u)\left(r_{u, j}-\bar{r}_{u}\right)
$$

where $\widehat{U}$ is a set of neighbours of user $a$.The coefficient $\alpha$ is an adjusted factor calculated by formula $\alpha=\frac{1}{\sum_{u \in U} \operatorname{sim}(a, u)}$.After all user preferences on items set are predicted, it is necessary to generate top- $\mathrm{N}$ items in recommendation.

In the consensus phase, the consensus-driven GRS generates group preference based on Choquet integral using formulae mentioned in Section 2.3. According to the Choquet-integral operation, defining a proper capacity function is crucial. According to the objective of this study, the capacity functions imply a fuzzy measure regarding to both additive total group users' satisfactions and balance between group users. We will discuss this in Section 3.2.

\subsection{The proposed Capacity Functions}

In this section, several new approaches for generation of capacity function are introduced. The first approach called the pure fairness-aware capacity function focuses on reducing the imbalance between members. The second approach named as the adjusted-fairness capacity function considers more influence of user interactions to user satisfaction in community.

\section{a. Pure Fairness-Aware Capacity Function}

For a group of users $g$, to select top-n items $I$ by Choquet integral-based aggregate operation, it is necessary to define a fuzzy measure on set of $|g|$ users. Remind that identification of all values belong to a fuzzy measure on set of $|g|$ users is a NP-hard problem. However, in the consensus phase using Choquet integral, for each item it is required to define capacity values on a linear extension on the poset of $g$. It means that we identify $|g|$ values only.

Herein, the pure fairness- aware approach considers the activeness of a user and his known interest in an itemset to define a capacity function. This approach adores the work presented in (Huynh et al., 2020). Considered a group of users $g$ as criteria for select an item. The capacity of one criterion is:

$$
\mu\left(u_{i}\right)=\left\{\begin{array}{c}
\frac{\omega\left(u_{i}\right)}{\sum_{u_{i} \in g} \omega\left(u_{i}\right)}, \text { if } \sum_{u_{i} \in g} \omega\left(u_{i}\right)<1 \\
\omega\left(u_{i}\right), \text { otherwise }
\end{array}\right.
$$

where $\omega\left(u_{i}\right)=\frac{\operatorname{count}\left(r_{u_{i}, j} \geq 0\right)}{|I|}, j \in[1,|I|]$, and capacity of subset of criteria $A \subset g$ is:

$\mu(A)=\sum_{u_{i} \in A} \mu\left(u_{i}\right)+\sigma(A)$

$$
\text { If } \mu(A)>1 \text { it is set to } 1 \text {. }
$$


Property 1: In case $\sigma(A)=0$, the capacity is an additive capacity and Choquet integral aggregation can be considered as the weighted sum aggregation.

Proof: Without loss of generality, assume that vector $r \in \mathbb{R}^{\mathrm{m}}, r=\left\{r_{u_{1}, i}, \ldots, r_{u_{m}, i}\right\}$ where $r_{u_{1}, i} \leq \ldots \leq r_{u_{m}, i}$. Then, the Choquet integral of a vector $r \in \mathbb{R}^{\mathrm{m}}$ with respect to a capacity $\mu$ is calculated by the following formula:

$$
\operatorname{pref}_{i}^{C Q}=\sum_{i=1}^{m}\left(r_{u_{i}, i}-r_{u_{i-1}, i}\right) \mu\left(\left\{u_{i}, u_{i+1}, \ldots, u_{m}\right\}\right),
$$

In which $r_{u_{0}, i}=0$.Remind that the capacity function $\mu$ is an additive capacity function, then, for each pair of subsets ' ,' $\in \mathrm{U}$ :

$$
\mu(A \cup B)=\mu(A)+\mu(B) .
$$

Therefore,

$$
\mu\left(\left\{u_{i}, u_{i+1}, \ldots, u_{m}\right\}\right)=\mu\left(\left\{u_{i}\right\}\right)+\mu\left(\left\{u_{i+1}, \ldots, u_{m}\right\}\right)=\sum_{j=i}^{m} \mu\left(\left\{u_{j}\right\}\right) .
$$

Thus, we have:

$$
\begin{aligned}
\operatorname{pref}_{i}^{C Q} & =\sum_{i=1}^{m}\left(\left(r_{u_{i}, i}-r_{u_{i-1}, i}\right) \sum_{j=i}^{m} \mu\left(\left\{u_{j}\right\}\right)\right), \\
\operatorname{pref}_{i}^{C Q} & =\sum_{i=1}^{m} r_{u_{i}, i} \mu\left(u_{i}\right),
\end{aligned}
$$

where $\mu\left(u_{i}\right)$ is the weight of each user in weight sum aggregation.In the pure fairness- aware approach, $\sigma(A) \neq 0$ is defined as follow.

$$
\sigma(A)=\sum_{u_{i} \in A} \omega^{\prime}\left(u_{i}\right)
$$

where:

$$
\omega^{\prime}\left(u_{i}\right)=\frac{\operatorname{count}\left(r_{u_{i}, j}>\text { aveRate }\right)-\operatorname{count}\left(r_{u_{i}, j}<\text { aveRate }\right)}{|I|}
$$

The item set $I$ contains all items rated by user $u_{i} \cdot \omega^{\prime}\left(u_{i}\right)$ presents a user interest in rated items.

\section{b. Adjusted Fairness Capacity Function}

In group activities, the interaction among group members indeed has strong impact on the satisfaction of members. With the same action, a person in a similar sub-group always has high satisfaction comparing with the case that he performs this action with another group of different favourites. Based on this fact, we propose an adjusted-fairness capacity function from the pure fairness-aware capacity function mentioned above. The newer approach adjusts capacity value of a group by the density of group.

$$
\sigma^{\prime}(A)=\frac{1}{\operatorname{dens}(A)} * \sigma(A)
$$


The density of group measures the similarity between all members based on Mahalanobis distance of all users.

$$
\operatorname{dens}(A)=\operatorname{Average}\left(\operatorname{dis}\left(u_{i}\right)\right), \forall u_{i} \in A
$$

Mahalanobis distance of a user $\operatorname{dis}\left(u_{i}\right)$ is the distance from that user to the center of group. The adjust capacity function presented in the above formulas means that a closer user group has a higher capacity value.

c. Proposed Consensus Mechanism Based on The Choquet Integral

Utilizing the capacity functions defined above, the preference of a group $g$ on an item $i$ in item set $I$ is calculated by the Choquet integral-based operation.

$$
\operatorname{pref}^{C Q}(g, i)=\sum_{n=1}^{|g|}\left(r_{n, i}^{\uparrow}-r_{n-1, i}^{\uparrow}\right) \mu\left(\Upsilon_{n}^{\uparrow}\right)
$$

where $r_{i}^{\uparrow}=\left\{r_{1, i}^{\uparrow}, r_{2, i}^{\uparrow}, \ldots, r_{m, i}^{\uparrow}\right\}, m=|g|$ is a permutation of the elements of vector present ratings of all user of group $g$ on item $i$, where $0=r_{0, i}^{\uparrow} \leq r_{1, i}^{\uparrow} \leq \ldots \leq r_{m, i}^{\uparrow}$, and the set $\Upsilon_{n}^{\uparrow}=\left\{u \in g, r_{u, i} \geq r_{n, i}^{\uparrow}\right\}=\left\{n^{\uparrow}, n+1^{\uparrow}, \ldots, m^{\uparrow}\right\}$ for $n<m$ and $\Upsilon_{m+1}^{\uparrow}=0$. When the preferences of a group on all items of candidate set $I$ are evaluated, the top-N recommendation for the group is generated trivially.

A brief version of Python-based pseudo-code for consensus algorithm is presented as above. This pseudo-code avoids some simple tasks of algorithm to make the main idea clearer.

\section{d. Complexity Analysis}

In the pseudocode above, the function get_permutation uses the selection sort algorithm to get the order of users based on user-item rating. This algorithm is simple and we do not describe in detail.

We can see that to calculate capacity values of a subset of user $A$, it requires to calculate $\omega(u)$ and $\omega^{\prime}(u)$, and a function that does this job has complexity as $\mathrm{O}(|A| \cdot|I|)$. To calculate the preference a group $g$ gives for item $i$ by Choquet integral-based aggregation, we have to calculate $m=|g|$ capacity values. A function does this task has complexity as $\mathrm{O}(|g| \cdot|I| \cdot|g|)$ (remind that $|A| \leq|g|)$.

The consensus phase has complexity $\mathrm{O}\left(\left|I^{\prime}\right| \cdot|g|^{2} \cdot|I| \cdot N\right)$. Because $|g|$ is often much smaller than $|I|$, this approach is close to Copeland Rule strategy and Borda Count strategy, and it is feasible in real-world applications. However, for adjusted fairness capacity function, foreach subset of user $A$, it requires to calculate $\operatorname{dens}(A)$ by Mahalanobis, and this function has high computing complexity. 
Table 3. Consensus algorithm

Choquet integral-based aggregation consensus algorithm

Inputs: user group $g$, set of candidate items $I^{\prime}$, rating matrix $R$ presents ratings of all users $U$ on items $I$.

Outputs: top-N prefered items for $g, N \leq\left|I^{\prime}\right|$

1. number_user $=|g|$

weight $=[$ None $] *$ number_user

weight_prime $=[\text { None }]^{*}$ number_user

for $\mathrm{i}$ in range (number_user):

get weight[i] by formula 1.19

get weight_prime[i] by formula 1.22

\# calculate the capacity value of a subset of a user permutation

def get_capacity(Permutation, Index):

cap_value $=0$

for $\bar{i}$ in range (Index, len(Permutation)):

cap_value $=$ cap_value + weigth $[\mathrm{i}]$

if (len(Permutation)-Index $>1$ ):

for $\mathrm{j}$ in range (Index, len(Permutation)):

cap_value $=$ cap_value + weigth_prime[j]

return cap_value

\# calculate group's preference on candidate items

2. number_candidate $=\left|I^{\prime}\right|$

group_preferences $=[\text { None }]^{*}$ number_candidate

for $\mathrm{i}$ in range (number_candidate):

3. user_permutation $=$ get_permutation $\left(g, I_{i}^{\prime}, R\right)$

\# calculate group's preference on an item by Choquet integral

group_item_pre $=0$

previous_rate $=0$

for $\mathrm{j}$ in range(number_user):

user $=$ user_permuation $[\mathrm{j}]$

substraction $=\mathrm{R}[$ user,i] - previous_rate

group_item_pre $+=$ substraction*get_capacity(user_permutation,j)

previous_rate $=\mathrm{R}$ [user,i]

group_preferences[i]= group_item_pre

\# return a recommendation

From vectorgroup_preferences return top-N best items.

\section{EXPERIMENTS}

\subsection{Environmental Data}

The literature review shows that there is no data that has an available explicit information of group users' activities. Therefore, in order to compare the proposed approach and the other strategies, the MovieLens-1Mdataset is used (Table 4).

Table 4. Dataset statistic information

\begin{tabular}{|l|l|l|l|}
\hline Dataset & Number of uses & Number of items & Number of ratings \\
\hline MovieLens 1M & 610 & 9742 & 100836 \\
\hline
\end{tabular}


The MovieLens-1M dataset is popularly used in researches about both recommender systems and group recommender systems. However, to use in GRS it requires a mechanism for generating groups of users.

Foreach user group $g_{i}$ the random sampling technique is used to select $\left|g_{i}\right|$ non-duplicated users from a dataset. In the testing process, the number of groups is fix, and the profiles of all groups are generated one time only for all experiment methods.

\subsection{Evaluation Metrics}

Recall that many consensus strategies can be used to build a consensus-driven GRS, and each strategy might have specific concern. There are different metrics developed to evaluate these concerns. However, to compare the performance of strategies mentioned in the above section and our proposal approach, two types of metrics are used, that are error and fairness metrics. We also introduced a new adjust fairness metric to extract the efficient of models on a specific new point of view.

\section{Error Metric}

Error metric is used to estimate error of group recommendations. This study use the mean absolute error (MAE)to calculate the error of a recommendation given to a group, and the average MAE of all group is the overall error of GRS model.

$$
\operatorname{MAE}(g, I)=\frac{\sum_{i \subset I}\left|r_{g, i}-\hat{r}_{g, i}\right|}{|I|}
$$

where $I$ is a set of items recommended to group $g$, and $r_{g, i}, \hat{r}_{g, i}$ are predicted group rating and real group rating, relatively. A group rating is calculated by average rating of all individuals in group.

$$
r_{g, i}=\frac{\sum_{u \subset g} r_{u, i}}{|g|}
$$

\section{Group Preference Metric}

Recall that in this study an important objective of GRS is searching for a solution that supports both fairness element and high group preference. Therefore, group preference metric is used and calculated by the following formula.

$$
\begin{aligned}
& \text { group_pref }_{-}(g, I)=\frac{\sum_{i \subset I} r_{g, i}}{|I|} \\
& \text { where } r_{g, i}=\frac{\sum_{u \in g} r_{u, i}}{|g|} \text { presents the average of user's preference on an item. }
\end{aligned}
$$

\section{Fairness Metrics}

To evaluate the fairness of a GRS model, we used two fairness metrics. 
- The first metric takes in to account the ratio of members who satisfy with the recommendation and group members. This metric can be calculated by formula (1.7). This measure can be extended to estimate the fairness of a recommendation of a set of items $I$ to a group by simple aggregation operation. The metric can vary from 0 to 1 and if the fairness value is equal to 1 it means that all users satisfy with recommendation.

- The second metric estimates the difference between group users' satisfaction. This metric can be calculated by formula (1.9) with an assumption that a user's rating presents a user's satisfaction. The metric can vary in interval $(-\infty, 1]$ and if the fairness value is equal to 1 it means that all users' satisfactions are equal.

\subsection{Result and Discussion}

This section presents the experimental results of the proposed models of Choquet integral-based aggregation with two capacity functions mentioned in Section 3.3. The performance of two models is compared with six common strategies used in consensus phase of GRS. According to the approach described in Section 4.1, a set of group sizes is set as $\{3,5,10,15,20\}$, and the number of group is 30. They are used to generate the testing groups by random sampling. The results of all models on MovieLens $1 \mathrm{M}$ dataset are presented in the following tables and figures.

Table 5. Average MAE values of the model vs. the number of recommended items

\begin{tabular}{|l|l|l|l|l|l|l|l|l|}
\hline Top-N & AUS & MS & LMS & MPS & AVS & CRS & CIS_1 & CIS_2 \\
\hline N=1 & 0.226 & 0.254 & $\mathbf{0 . 4 6 4}$ & 0.240 & 0.409 & 0.224 & 0.336 & 0.319 \\
\hline $\mathrm{N}=2$ & 0.219 & 0.252 & $\mathbf{0 . 4 2 3}$ & 0.247 & 0.377 & 0.217 & 0.264 & 0.251 \\
\hline $\mathrm{N}=3$ & 0.201 & 0.217 & $\mathbf{0 . 4 1 1}$ & 0.236 & 0.410 & 0.197 & 0.251 & 0.248 \\
\hline $\mathrm{N}=4$ & 0.185 & 0.207 & $\mathbf{0 . 3 9 4}$ & 0.236 & 0.382 & 0.184 & 0.243 & 0.244 \\
\hline $\mathrm{N}=5$ & 0.173 & 0.208 & $\mathbf{0 . 3 8 5}$ & 0.225 & 0.356 & 0.175 & 0.257 & 0.258 \\
\hline $\mathrm{N}=6$ & 0.171 & 0.207 & $\mathbf{0 . 3 7 8}$ & 0.204 & 0.374 & 0.171 & 0.265 & 0.268 \\
\hline $\mathrm{N}=7$ & 0.171 & 0.201 & 0.363 & 0.190 & $\mathbf{0 . 3 7 0}$ & 0.173 & 0.257 & 0.262 \\
\hline $\mathrm{N}=8$ & 0.163 & 0.200 & 0.359 & 0.187 & $\mathbf{0 . 3 6 6}$ & 0.163 & 0.274 & 0.278 \\
\hline $\mathrm{N}=9$ & 0.172 & 0.203 & $\mathbf{0 . 3 5 3}$ & 0.187 & 0.352 & 0.172 & 0.277 & 0.281 \\
\hline $\mathrm{N}=10$ & 0.178 & 0.202 & 0.338 & 0.203 & $\mathbf{0 . 3 5 2}$ & 0.178 & 0.282 & 0.285 \\
\hline
\end{tabular}

Table 6. Average MAE values of model vs. group size

\begin{tabular}{|l|l|l|l|l|l|l|l|l|}
\hline Group size & AUS & MS & LMS & MPS & AVS & CRS & CIS_1 & CIS_2 \\
\hline 3 & 0.095 & 0.081 & 0.092 & 0.071 & 0.080 & 0.095 & $\mathbf{0 . 1 8 2}$ & $\mathbf{0 . 1 8 2}$ \\
\hline 5 & 0.173 & 0.197 & $\mathbf{0 . 3 8 6}$ & 0.242 & 0.380 & 0.171 & 0.220 & 0.220 \\
\hline 10 & 0.197 & 0.222 & $\mathbf{0 . 4 2 4}$ & 0.272 & 0.421 & 0.197 & 0.259 & 0.259 \\
\hline 15 & 0.224 & 0.249 & 0.469 & 0.255 & $\mathbf{0 . 4 9 0}$ & 0.224 & 0.290 & 0.300 \\
\hline 20 & 0.240 & 0.327 & $\mathbf{0 . 5 6 4}$ & 0.238 & 0.503 & 0.240 & 0.402 & 0.385 \\
\hline
\end{tabular}


Error metric presents performance of user rating prediction algorithm in recommendation phase of GRS. As described in section 3.2, the accuracy of this phase depends mostly on user-based collaborative filtering algorithm. Strategies used in the consensus phase have little effect on this metric. Therefore, in this section, we do not compare the accuracy of different strategies. We validate the performance of all strategies by different group sizes and the number of items in recommendation of GRS.

Tables $\mathbf{2}$ and $\mathbf{3}$ show that for all strategies including the Choquet integral-based aggregation, the MAE values tend to decrease when the number of recommended items increases. This error increases monotonically with the size of group.

The first fairness metric presents the ratio of users satisfied with recommendation and group's size. In this study, a user is considered as satisfied user if he rates an item equal or greater than a threshold, $t h=3.5$. This threshold means that a satisfied user is a user who like recommended item. Table 4 shows that the approval voting strategy and least misery strategy are two best models regarding first type of fairness.

Table 7. Average ratio of users satisfied and group's size groups'

\begin{tabular}{|l|l|l|l|l|l|l|l|l|}
\hline Top-N & AUS & MS & LMS & MPS & AVS & CRS & CIS_1 & CIS_2 \\
\hline $\mathrm{N}=1$ & 0.701 & 0.760 & 0.820 & 0.651 & $\mathbf{0 . 8 5 1}$ & 0.701 & 0.694 & 0.675 \\
\hline $\mathrm{N}=2$ & 0.721 & 0.757 & 0.831 & 0.642 & $\mathbf{0 . 8 4 6}$ & 0.721 & 0.687 & 0.672 \\
\hline $\mathrm{N}=3$ & 0.725 & 0.747 & 0.820 & 0.640 & $\mathbf{0 . 8 5 2}$ & 0.725 & 0.681 & 0.676 \\
\hline $\mathrm{N}=4$ & 0.718 & 0.752 & 0.813 & 0.636 & $\mathbf{0 . 8 3 4}$ & 0.718 & 0.676 & 0.675 \\
\hline $\mathrm{N}=5$ & 0.716 & 0.753 & 0.800 & 0.627 & $\mathbf{0 . 8 3 1}$ & 0.716 & 0.681 & 0.680 \\
\hline $\mathrm{N}=6$ & 0.715 & 0.742 & 0.797 & 0.624 & $\mathbf{0 . 8 2 7}$ & 0.715 & 0.680 & 0.680 \\
\hline $\mathrm{N}=7$ & 0.715 & 0.742 & 0.787 & 0.622 & $\mathbf{0 . 8 1 9}$ & 0.715 & 0.680 & 0.682 \\
\hline $\mathrm{N}=8$ & 0.706 & 0.740 & 0.782 & 0.618 & $\mathbf{0 . 8 1 1}$ & 0.706 & 0.686 & 0.688 \\
\hline $\mathrm{N}=9$ & 0.701 & 0.734 & 0.779 & 0.615 & $\mathbf{0 . 8 0 7}$ & 0.701 & 0.689 & 0.690 \\
\hline $\mathrm{N}=10$ & 0.702 & 0.730 & 0.775 & 0.618 & $\mathbf{0 . 8 0 7}$ & 0.702 & 0.690 & 0.690 \\
\hline
\end{tabular}

Recall that the main objective of GRS is to find the good solution regarding both increasing average group's preference and reducing imbalance between group users. Herein, we investigate these two important elements in details. Table $\mathbf{6}$ shows the difference of models on average groups' preference. It is trivial to get that AUS has the highest groups' preference because it uses the maximize additive users' preference. GRS with Copeland rule strategy has the same performance with AUS. The two proposal algorithms are close to the AUS, and they are better than other strategies.

Figures 2-4 show the performance of all models in three difference group sizes. It is clearly shown the convergence of groups' preference of all models when the number of recommended items increases.

Variance fairness metric is used to present imbalance between group users' ratings. Figures 5-7 show the performance of all models in three difference group sizes.

It can be seen that the Least misery strategy (LMT) and Approval voting strategy (AVT) have highest fairness element. However, these strategies cause the lowest average groups' preference. Most pleasure strategy(MPT) gives lowest fairness solution in all cases, and it has low average groups' preference too. Additive utilitarian strategy (AUT) and Copeland rule strategy (CRT) give highest average group's preference but they have low fairness. GRSs with two proposal Choquet integralbased aggregation are not outperform other models in any of both sides: average group's preference 
and fairness element. Though, it is easy to see that these two proposal models are balance between both objectives. The GRS with Multiplicative strategy (MT) is competent but its performance reduces when number of recommended items or group size increases.

Table 8. Average groups' rating vs number of recommended items

\begin{tabular}{|l|l|l|l|l|l|l|l|l|}
\hline Top-N & AUS & MS & LMS & MPS & AVS & CRS & CIS_1 & CIS_2 \\
\hline N=1 & $\mathbf{4 . 7 1 7}$ & 4.694 & 4.373 & 4.384 & 4.412 & 4.717 & 4.556 & 4.560 \\
\hline N=2 & $\mathbf{4 . 6 8 9}$ & 4.670 & 4.350 & 4.404 & 4.383 & 4.689 & 4.536 & 4.537 \\
\hline N=3 & $\mathbf{4 . 6 6 9}$ & 4.658 & 4.339 & 4.387 & 4.357 & 4.669 & 4.514 & 4.516 \\
\hline N=4 & $\mathbf{4 . 6 5 2}$ & 4.645 & 4.337 & 4.396 & 4.350 & 4.652 & 4.503 & 4.505 \\
\hline N=5 & $\mathbf{4 . 6 3 7}$ & 4.628 & 4.331 & 4.397 & 4.349 & 4.637 & 4.481 & 4.483 \\
\hline N=6 & $\mathbf{4 . 6 2 5}$ & 4.615 & 4.327 & 4.401 & 4.342 & 4.625 & 4.465 & 4.467 \\
\hline N=7 & $\mathbf{4 . 6 1 4}$ & 4.605 & 4.313 & 4.402 & 4.341 & 4.614 & 4.451 & 4.453 \\
\hline N=8 & $\mathbf{4 . 6 0 0}$ & 4.594 & 4.301 & 4.398 & 4.339 & 4.600 & 4.442 & 4.444 \\
\hline N=9 & $\mathbf{4 . 5 8 6}$ & 4.578 & 4.292 & 4.390 & 4.335 & 4.586 & 4.433 & 4.433 \\
\hline N=10 & $\mathbf{4 . 5 7 2}$ & 4.564 & 4.279 & 4.378 & 4.331 & 4.572 & 4.420 & 4.420 \\
\hline
\end{tabular}

Figure 2. Average groups' rating with group size 3

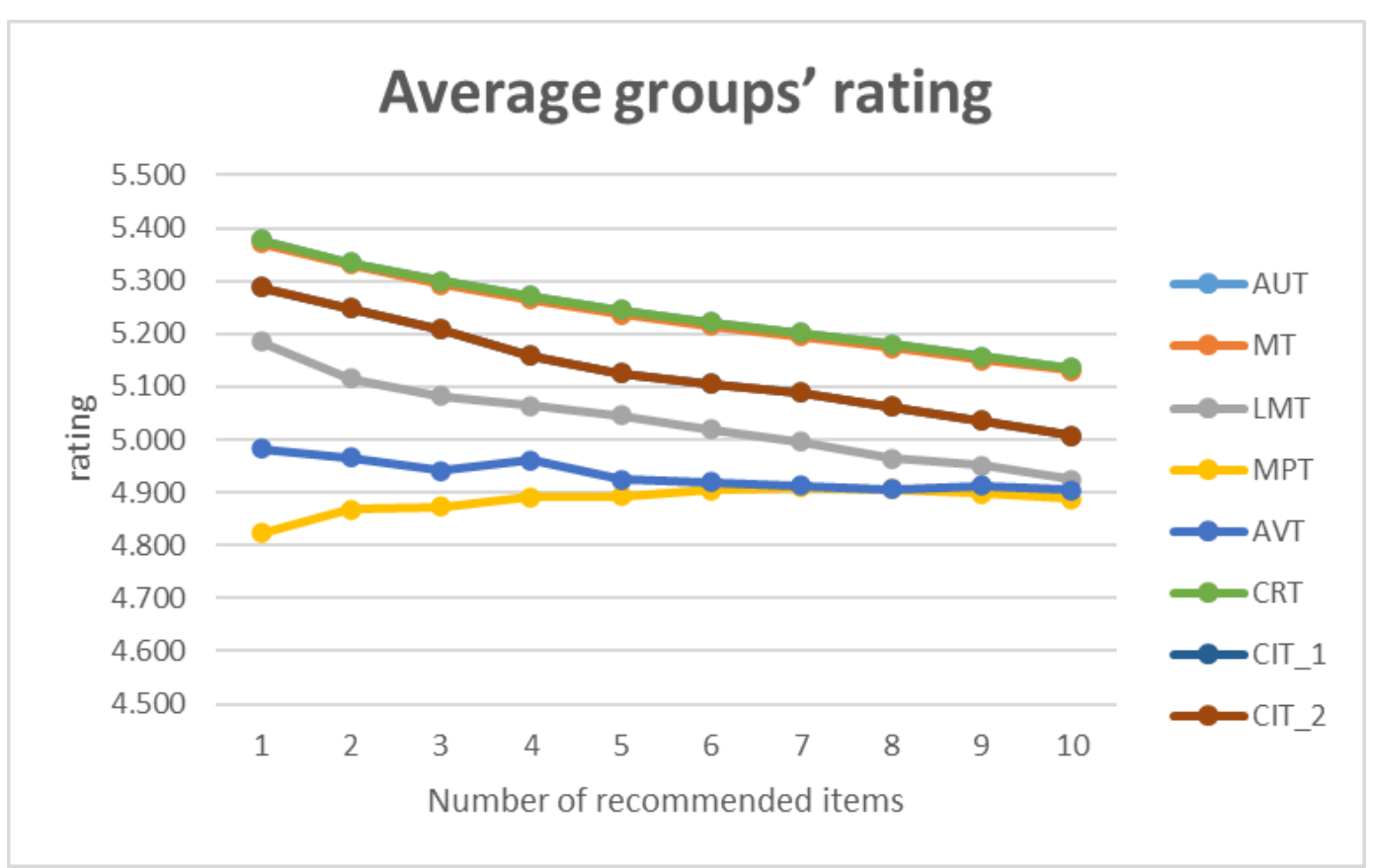


Figure 3. Average groups' rating with group size 10

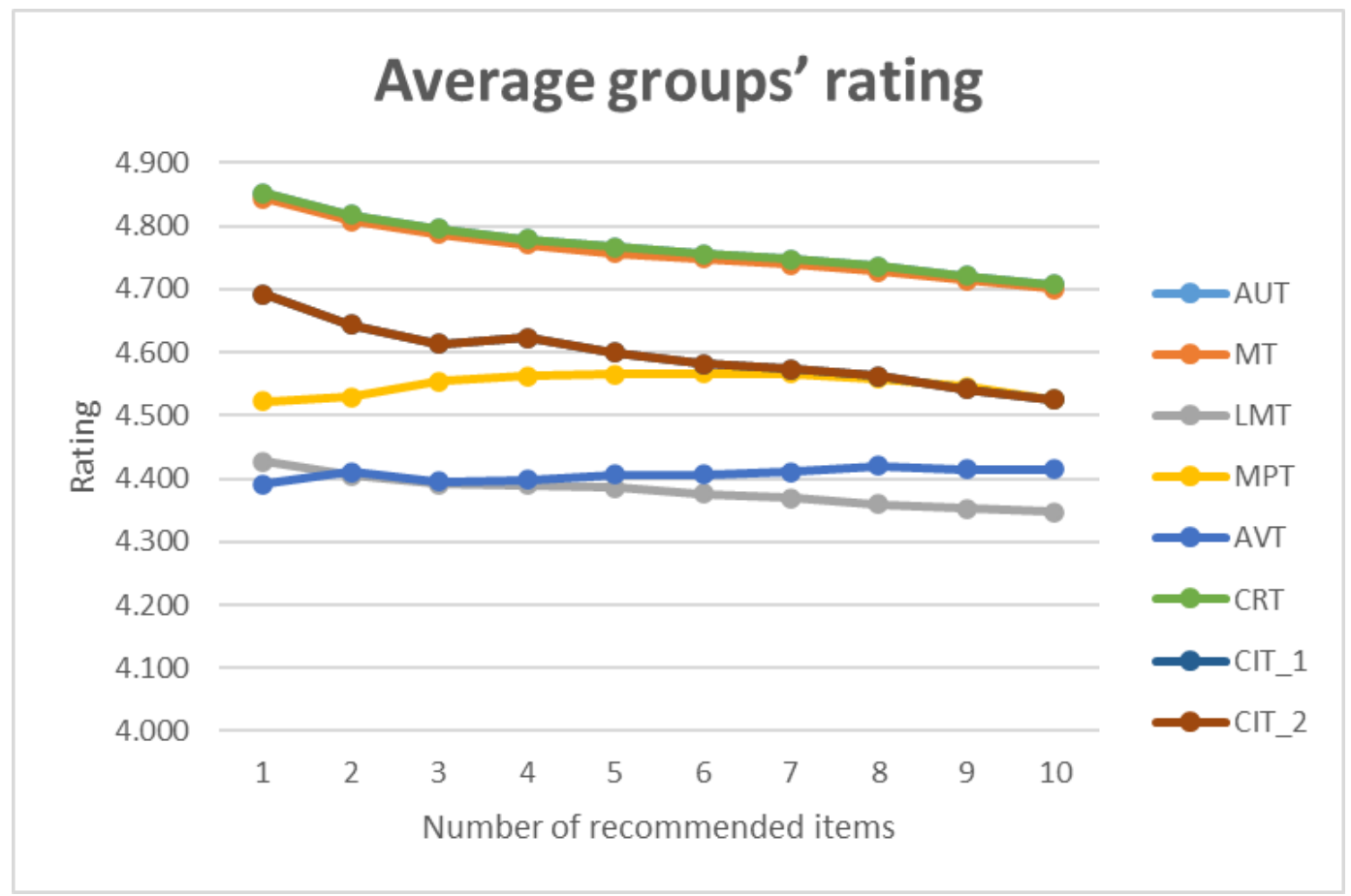

Figure 4. Average groups' rating with group size 20

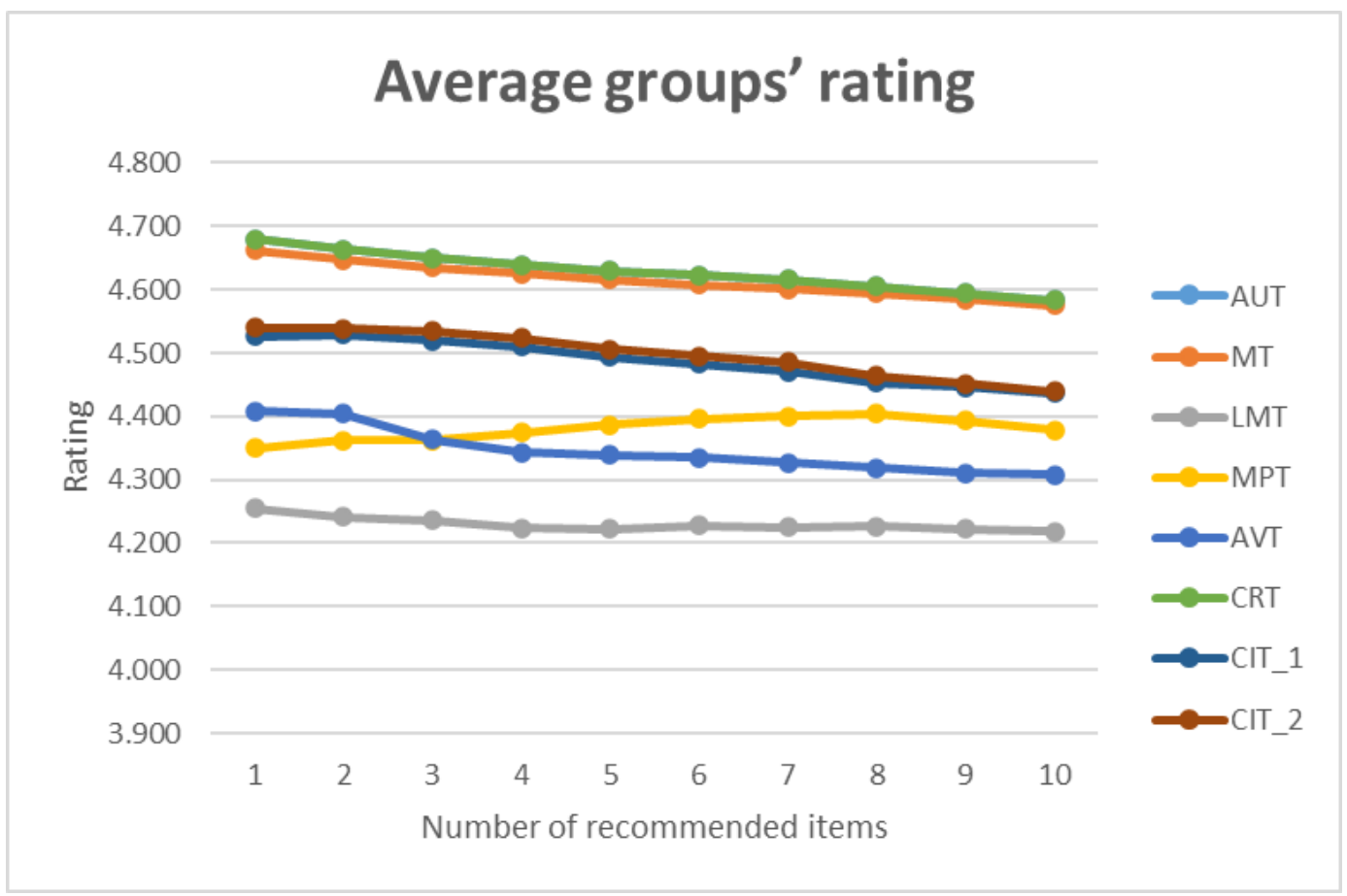


Figure 5. Fairness of GRS with group size 3

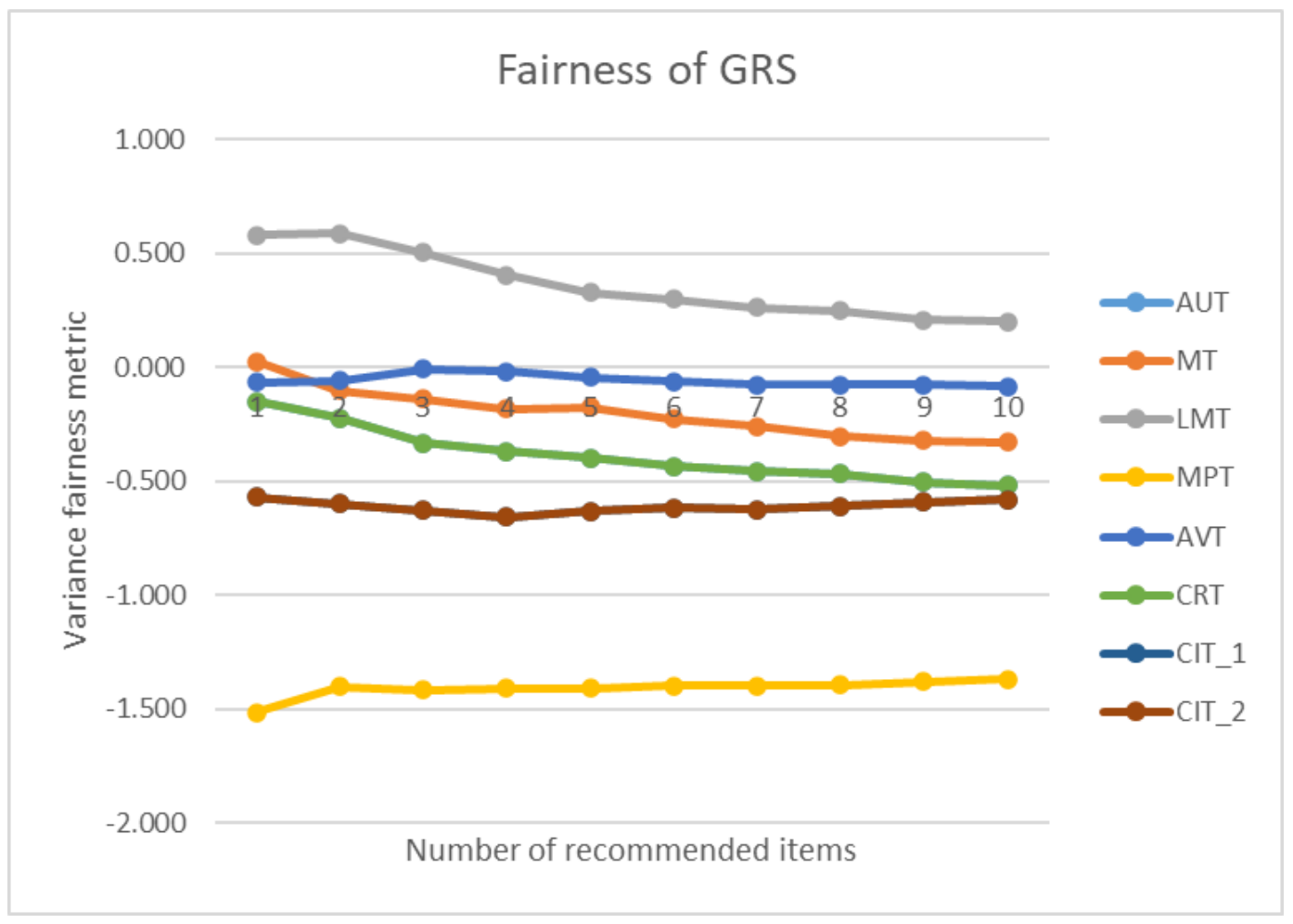

Figure 6. Fairness of GRS with group size 10

\section{Fairness of GRS}

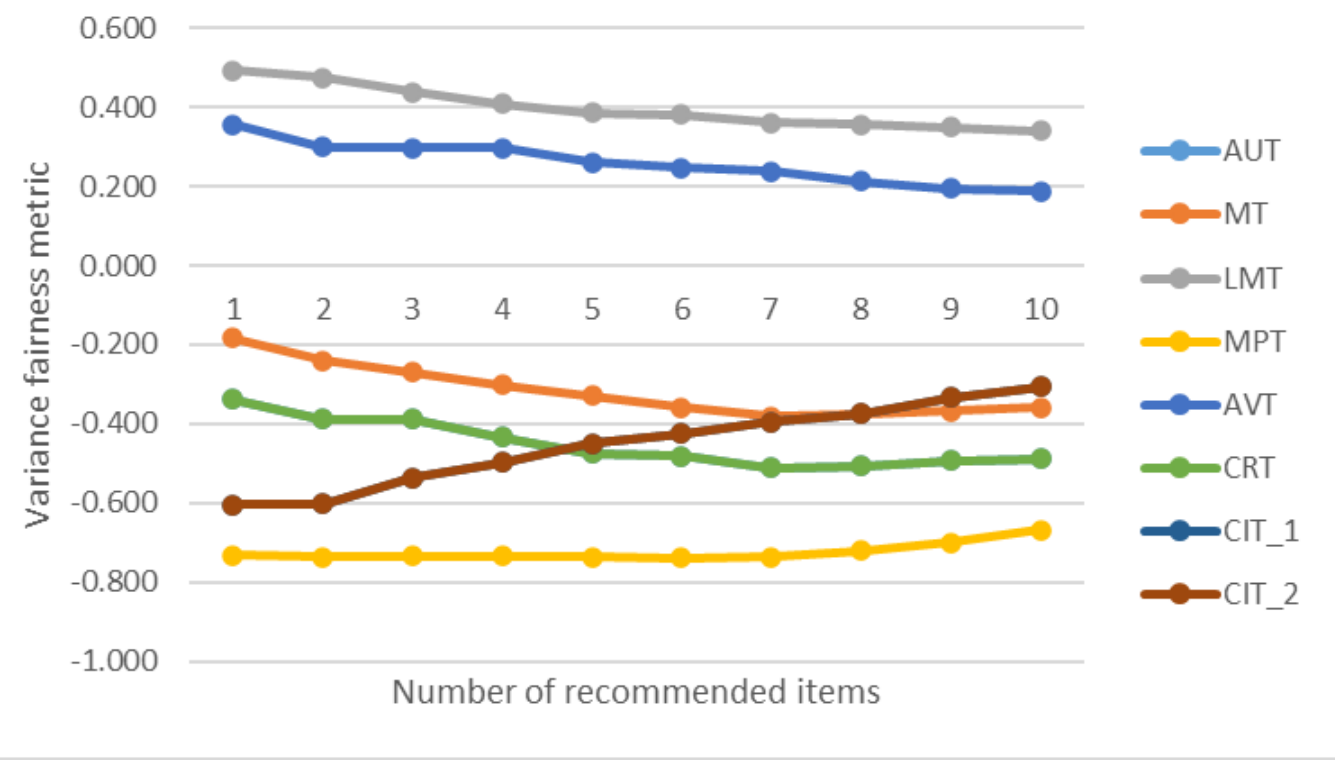




\section{Fairness of GRS}

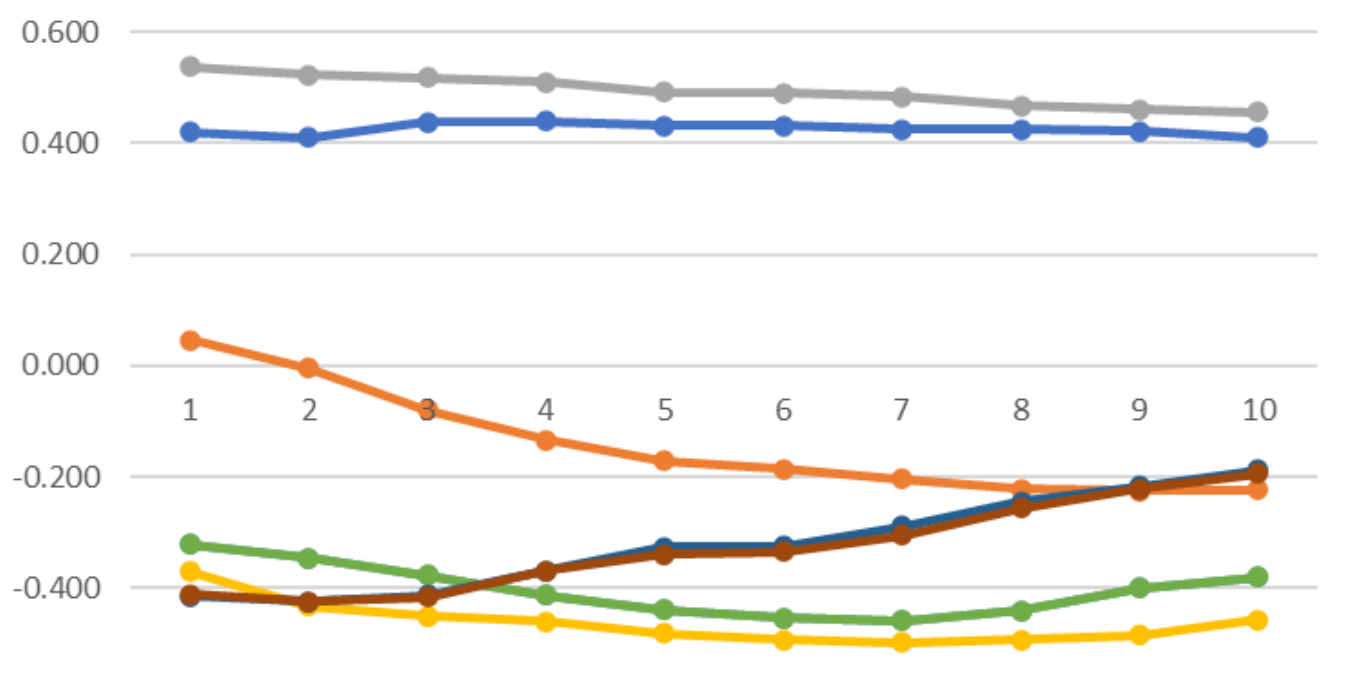

$-0.600$

$\longrightarrow \mathrm{AUT} \longrightarrow \mathrm{MT} \longrightarrow \mathrm{LMT} \longrightarrow \mathrm{MPT} \longrightarrow \mathrm{AVT} \longrightarrow \mathrm{CRT} \longrightarrow \mathrm{CIT}$ 1 $\longrightarrow$ CIT_2

\section{CONCLUSION}

Along with additive group users' preference, fairness is an important perspective in joint activities and therefore it is an important criterion in group recommender systems. This paper investigated the fairness of consensus-driven GRSs with different strategies and proposed a new approach based on Choquet integral for consensus mechanism. New consensus mechanism extends the search space of weighted sum aggregation including additive utilitarian strategy operation to reach more balance recommendation theoretically. They also promote a mechanism to handle an important point in GRS that is effect of users' interaction.

Besides, experiments were designed to investigate the performance of the new proposed approaches against the existing strategies of consensus mechanism. The selected dataset, MovieLens $1 \mathrm{M}$, does not contain explicit information of user groups therefore a random sampling method was used to generate simulated groups. Error metric, fairness metrics and group preference metric are used to investigate different sides of GRSs. Therein, content of fairness is variable due to application domains. Therefore, this study used two different existing fairness metrics but focuses solely on the variance fairness because it shows imbalance between group's users.

The experimental results show the advantage of Choquet integral-based aggregate operation in consensus-driven group recommender system. While AUT and CRP seem to be extreme solutions for applications requiring optimum group's preference, LMT and AVT are extreme solutions for circumstances that need optimum fairness element. In opposite, the proposed models balance both sides above. They generated high additive group users' preference and good fairness recommendations. This advantage is significant when GRSs have to recommend a high number of items or services to large group of users. 
The future work will focus on improving computing time of GRS using adjust fairness capacity function and investigate more on effect of users' interaction under this GRS context (Huynh\& Phan et al., 2020).

\section{ACKNOWLEDGMENT}

- This work was supported by the Institute of Information Technology, Vietnam Academy of Science and Technology, under Project CS21.06.

- The corresponding author of this paper is Dr. Nguyen Nhu Son (email: nnson@ioit.ac.vn) 


\section{REFERENCES}

Banda, L., Singh, K., Abdel-Basset, M., Thong, P. H., Huynh, H. X., \& Taniar, D. (2020). Recommender Systems Using Collaborative Tagging. International Journal of Data Warehousing and Mining, 16(3), 183-200. doi:10.4018/IJDWM.2020070110

Cantador, I., \& Castells, P. (2012). Group recommender systems: new perspectives in the social web. In Recommender systems for the social web (pp. 139-157). Springer. doi:10.1007/978-3-642-25694-3_7

Castro, J., Quesada, F. J., Palomares, I., \& Martinez, L. (2015). A consensus-driven group recommender system. International Journal of Intelligent Systems, 30(8), 887-906. doi:10.1002/int.21730

Combarro, E. F., DiAz, I., \& Miranda, P. (2013). On random generation of fuzzy measures. Fuzzy Sets and Systems, 228, 64-77. doi:10.1016/j.fss.2012.09.006

Da'u, A., \& Salim, N. (2020). Recommendation system based on deep learning methods: A systematic review and new directions. Artificial Intelligence Review, 53(4), 2709-2748. doi:10.1007/s10462-019-09744-1

Dara, S., Chowdary, C. R., \& Kumar, C. (2020). A survey on group recommender systems. Journal of Intelligent Information Systems, 54(2), 271-295. doi:10.1007/s10844-018-0542-3

Felfernig, A., Boratto, L., Stettinger, M., \& Tkalčič, M. (2018). Evaluating group recommender systems. In Group recommender systems (pp. 59-71). Springer. doi:10.1007/978-3-319-75067-5_3

Huynh, H. X., Cu, G. N., Huynh, T. M., \& Luong, H. H. et al. (2020). Recommender Systems Based on Resonance Relationship of Criteria With Choquet Operation. International Journal of Data Warehousing and Mining, 16(4), 44-62. doi:10.4018/IJDWM.2020100103

Huynh, H. X., Phan, N. Q., Pham, N. M., Pham, V. H., Abdel-Basset, M., \& Ismail, M. (2020). Context-Similarity Collaborative Filtering Recommendation. IEEE Access: Practical Innovations, Open Solutions, 8, 33342-33351. doi:10.1109/ACCESS.2020.2973755

Kaya, M., Bridge, D., \& Tintarev, N. (2020, September). Ensuring fairness in group recommendations by ranksensitive balancing of relevance. In Fourteenth ACM Conference on Recommender Systems (pp. 101-110). doi:10.1145/3383313.3412232

Kuhlman, C., \& Rundensteiner, E. (2020). Rank aggregation algorithms for fair consensus. Proceedings of the VLDB Endowment International Conference on Very Large Data Bases, 13(12), 2706-2719. doi:10.14778/3407790.3407855

Lerche, D., \& Sørensen, P. B. (2003). Evaluation of the ranking probabilities for partial orders based on random linear extensions. Chemosphere, 53(8), 981-992. doi:10.1016/S0045-6535(03)00558-7 PMID:14505721

Lust, T. (2015). Choquet integral versus weighted sum in multicriteria decision contexts. International Conference on Algorithmic DecisionTheory, 288-304. doi:10.1007/978-3-319-23114-3_18

Serbos, D., Qi, S., Mamoulis, N., Pitoura, E., \& Tsaparas, P. (2017). Fairness in package-to-group recommendations. Proceedings of the 26th International Conference on World Wide Web, 371-379. doi:10.1145/3038912.3052612

Wang, W., Zhang, G., \& Lu, J. (2017). Hierarchy visualization for group recommender systems. IEEE Transactions on Systems, Man, and Cybernetics. Systems, 49(6), 1152-1163. doi:10.1109/TSMC.2017.2699690

Xiao, L., Min, Z., Yongfeng, Z., Zhaoquan, G., Yiqun, L., \& Shaoping, M. (2017). Fairness-aware group recommendation with pareto-efficiency. Proceedings of the Eleventh ACM Conference on Recommender Systems, 107-115. doi:10.1145/3109859.3109887

Yin, H., Wang, Q., Zheng, K., Li, Z., \& Zhou, X. (2020). Overcoming data sparsity in group recommendation. IEEE Transactions on Knowledge and Data Engineering, 1. Advance online publication. doi:10.1109/ TKDE.2020.3023787 
Cu Nguyen Giap was born in 1984 in Phutho province, Vietnam. He received BSc. degree of Information Technology at Hanoi University of Technology in 2007. In 2012, he received MSc. degree of Computer Science in Vrije Universiteit Brussels. He is a lecturer in Faculty of Management Information System \& E-commerce in Thuongmai University, Vietnam. He has published several articles in the domain of applying computer science. His research interests include Soft-computing, Neutral network, Parallel Algorithms, and Applying data mining and machine learning algorithms.

Nguyen Nhu Son obtained the PhD degree on Computer Science at The University of Queensland - Australia in 2007. Dr. Son has worked as senior researcher and Head of Department at the Institute of Information Technology - Vietnam Academy of Science and Technology since 2008. His major fields include Artificial Intelligence, Data Mining, Soft Computing, Fuzzy Computing.

Nguyen Long Giang obtained the PhD degree on Math Fundamentals for Informatics at Institute of Information Technology (IoIT), Vietnam Academy of Science and Technology (VAST) in 2012. He has been promoted to Associate Professor in Information Technology since 2017. Dr. Giang worked as senior researcher and Head of Department at the IolT, VAST during 2009 - 2018. His major fields include Artificial Intelligence, Data Mining, Soft Computing, Fuzzy Computing, Rough Sets, Fuzzy Rough Sets.

Hoang Thi Minh Chau is a lecturer at University of Economics - Technology for Industries (UNETI), Hanoi, Vietnam. She has more than 15 years of teaching. Her research is data mining and fuzzy logic.

Tran Manh Tuan received the Bachelor on Applied Mathematics and Informatics at Hanoi University of Science and Technology in 2003 and Master degree on Computer Science at Thainguyen University in 2007. He got Doctoral degree in July, 2017. Now, he is working as a lecturer at Faculty of Computer Science and Engineering, Thuyloi University. His researches focus on Artificial Intelligence, Data Mining, Soft Computing, Fuzzy Computing.

Le Hoang Son is a Senior Researcher of Department of Multimedia and Virtual Reality, VNU Information Technology Institute. His major fields include Artificial Intelligence, Data Mining, Soft Computing, Fuzzy Computing, Fuzzy Recommender Systems, Geographic Information System. Dr. Son is an Associate Editor of Journal of Intelligent \& Fuzzy Systems (SCIE), IEEE Access (SCIE), Data Technologies and Applications (SCIE), International Journal of Data Warehousing and Mining (SCIE), Neutrosophic Sets and Systems (ESCI), Vietnam Research and Development on Information and Communication Technology, VNU Journal of Science: Computer Science and Communication Engineering, Frontiers in Artificial Intelligence. He serves as Editorial Board of Applied Soft Computing (SCIE), PLOS ONE (SCIE), International Journal of Web and Grid Services (SCIE), International Journal of Ambient Computing and Intelligence (ESCI), and Vietnam Journal of Computer Science and Cybernetics. 